\title{
A proposal for a standardized bacterial taxonomy based on genome phylogeny
}

Donovan H. Parks ${ }^{1}$, Maria Chuvochina ${ }^{1}$, David W. Waite ${ }^{1}$, Christian Rinke ${ }^{1}$, Adam

Skarshewski $^{1}$, Pierre-Alain Chaumeil ${ }^{1}$, Philip Hugenholtz ${ }^{1 *}$

5

${ }^{1}$ Australian Centre for Ecogenomics, School of Chemistry and Molecular Biosciences, The University of Queensland, QLD 4072, Australia

* Correspondence to: p.hugenholtz@uq.edu.au

\begin{abstract}
Taxonomy is a fundamental organizing principle of biology, which ideally should be based on evolutionary relationships. Microbial taxonomy has been greatly restricted by the inability to 15 obtain most microorganisms in pure culture and, to a lesser degree, the historical use of phenotypic properties as the basis for classification. However, we are now at the point of obtaining genome sequences broadly representative of microbial diversity by using cultureindependent techniques, which provide the opportunity to develop a comprehensive genomebased taxonomy. Here we propose a standardized bacterial taxonomy based on a concatenated 20 protein phylogeny that conservatively removes polyphyletic groups and normalizes ranks based on relative evolutionary divergence. From 94,759 bacterial genomes, 99 phyla are described including six major normalized monophyletic units from the subdivision of the Proteobacteria, and amalgamation of the Candidate Phyla Radiation into the single phylum Patescibacteria. In total, $73 \%$ of taxa had one or more changes to their existing taxonomy.
\end{abstract}




\section{Introduction}

The rapid expansion of sequenced microbial genomes in the past few years now makes it feasible to construct a detailed taxonomy based on genome sequences (Segata et al., 2013; Hugenholtz et al., 2016; Garrity et al., 2016; Yoon et al., 2017). Microbial taxonomy, the classification of microorganisms, is essential for accurately describing microbial diversity and providing a common language for communicating scientific results (Godfray 2002). Sequence-based phylogenetic trees provide a natural framework for defining a taxonomy that takes into account evolutionary relationships and differing rates of evolution. Current microbial taxonomy is often inconsistent with evolutionary relationships as many taxa circumscribe polyphyletic groupings

35 (McDonald et al., 2012). This is partly attributable to historical phenotype-based classification as exemplified by the clostridia where microorganisms sharing morphological similarities have been erroneously classified in the genus Clostridium (Yutin \& Galparin, 2013; Beiko 2015). Modern microbial taxonomy is primarily guided by $16 \mathrm{~S}$ rRNA relationships and such discrepancies are observable in 16S rRNA gene trees (McDonald et al., 2012; Yilmaz et al.,

40 2014), but most have not been corrected due to the scale of the task and the lengthy process of formally reclassifying microorganisms (Yarza et al., 2014).

A second less obvious issue with the existing sequence-based microbial taxonomy is the uneven application of ranks across the tree. Regions that are the subject of intense study tend to be split 45 into more taxa than other parts of the tree with equivalent phylogenetic depth, for example, the family Enterobacteriaceae (comprising dozens of genera) is equivalent to single genera in other parts of the tree such as Bacillus (Abbott \& Janda, 2006). Conversely, understudied groups are often lumped together, for example, the phylum Synergistetes is currently represented by a single family (Jumas-Bilak et al., 2009) that would constitute multiple family level groupings in more

50 intensively studied parts of the tree. A recent study by Yarza et al. (2014) proposed standardizing taxonomic ranks using 16S rRNA sequence identity thresholds and showed a high degree of discordance between these thresholds and existing taxonomy.

Current microbial taxonomies based on $16 \mathrm{~S}$ rRNA gene relationships have a number of 55 limitations including low phylogenetic resolution at the highest and lowest ranks (Case et al., 2007; Janda et al., 2007), missing diversity due to primer mismatches (Schulz et al., 2017), and 
PCR-produced chimeric sequences that can corrupt tree topologies by drawing together disparate groups (DeSantis et al., 2006). Trees inferred from the concatenation of single-copy verticallyinherited proteins provide higher resolution than those obtained from a single phylogenetic marker gene (Ciccarelli et al., 2006; Thiergart et al., 2014), and are increasingly representative of microbial diversity as culture-independent techniques are now producing thousands of metagenome-assembled genomes (MAGs) from diverse microbial communities (Brown et al., 2015; Anantharaman et al., 2016; Parks et al., 2017). Despite some caveats of their own (Thiergart et al., 2014), concatenated protein trees have been extensively used in the literature

65 (Tonini et al., 2015; Brown et al., 2015; Hug et al., 2016), are largely congruent with 16S rRNA gene trees (Brown et al., 2001; Ciccarelli et al., 2006), and have been proposed as the best basis for a reference bacterial phylogeny (Lang et al., 2013).

Here we propose a bacterial taxonomy based on a phylogeny inferred from the concatenation of 120 ubiquitous, single-copy proteins that covers 94,759 bacterial genomes, including 13,636 (14.4\%) from uncultured organisms (metagenome-assembled or single-cell genomes). Taxonomic groups within this classification describe monophyletic lineages of similar phylogenetic depths after normalizing for lineage-specific rates of evolution. This taxonomy, which we have called the GTDB taxonomy, is publicly available at the Genome Taxonomy

75 Database website (http://gtdb.ecogenomic.org).

\section{Results}

\section{Deriving the GTDB taxonomy}

A dataset comprising 87,106 bacterial genomes was obtained from RefSeq/GenBank release 80 (Haft et al., 2017) and augmented with 11,603 MAGs obtained from Sequence Read Archive metagenomes according to the approach of Parks et al. (2017). After removal of 2,482 of these genomes based on a completeness/contamination threshold and 1,468 based on a multiple sequence alignment threshold, the resulting 94,759 were dereplicated to remove highly similar genomes, with high-quality reference material being retained as representatives where possible (see Methods). Nearly $40 \%(8,559)$ of the dereplicated dataset of 21,943 genomes represent 85 uncultured organisms reflecting the microbial diversity currently being revealed by culture- 
independent techniques (Brown et al., 2015; Anantharaman et al., 2016; Parks et al., 2017). A bacterial genome tree was inferred from the dereplicated dataset using a concatenated alignment of 120 ubiquitous, single-copy proteins (subsequently referred to as 'bac120'; Parks et al., 2017) comprising a total of 34,744 columns after trimming 1,021 represented in $<50 \%$ of taxa and

905,390 with an amino acid consensus $<25 \%$. The bac120 dataset represents $\sim 4 \%$ of an average bacterial genome and is comparable to other bacterial domain marker sets (Dupont et al., 2012; Wu et al., 2013a).

Having inferred the concatenated protein phylogeny, we annotated the tree with group names using the National Center for Biotechnology Information (NCBI) taxonomy for the public genomes standardized to seven ranks (see Methods; Federhen 2012). Taxon names were overwhelmingly assigned to interior nodes with high bootstrap support $(99.7 \% \pm 2.9 \%)$ to ensure taxonomic stability. However, a few poorly supported nodes $(<70 \%)$ in the bac120 tree were assigned names based on independent analyses or to preserve widely used existing classifications

100 (Supp. Table 1; see Firmicutes example below). Since over a third of the dataset represents uncultured organisms, a substantial part of the tree was not effectively annotated using the NCBI genome taxonomy. Therefore, 16S rRNA gene sequences present in the MAGs were classified against the Greengenes 2013 (McDonald et al., 2012) and SILVA v123.1 (Yilmaz et al., 2014) taxonomies to provide additional taxonomic identifiers. Using a set of criteria to ensure accurate 105 mapping between 16S rRNA and MAG sequences (see Methods), 74 groups lacking cultured representatives were labeled with 16S rRNA-based names, including well-recognized clades such as SAR202 (Giovannoni et al., 1996), WS6 (Dojka et al., 1998) and ACK-M1 (Zwart et al., 2003) (Supp. Table 2). We term all such alphanumeric names non-standard placeholders to be replaced with standard validated names in due course. Curation of the taxonomy then involved 110 two main tasks, (i) removal of polyphyletic groups and (ii) normalization of ranks based on relative evolutionary divergence.

\section{Removal of polyphyletic groups}

Twenty phyla and 25 classes as defined by the NCBI taxonomy could not be reproducibly 115 resolved as monophyletic in the bootstrapped bac120 tree (Supp. Table 3). Most of these were the result of a small number of misclassified genomes; however, some taxa appear to be truly 
polyphyletic including well-known lineages such as the Firmicutes and Proteobacteria (Supp. Table 3). Instability of the Firmicutes has been previously noted, primarily as a result of the Tenericutes and/or Fusobacteria moving into or out of the group (Wolf et al., 2004; Hug et al.,

120 2016). In this prominent case, we chose to preserve the existing classification until more in-depth phylogenetic analyses are performed to resolve the issue (see rationale below). Other poorlysupported lineages such as the Proteobacteria, which is widely reported as polyphyletic using the 16S rRNA gene (Lonergan et al., 1996; McDonald et al., 2012) and protein markers (Beiko, 2011; Zhang \& Sievert, 2014; Vanwonterghem et al., 2016), were conservatively divided into

125 stable monophyletic groups. Where possible, polyphyletic taxa containing the nomenclature type retained the name, and all other groups were renamed according to the International Code of Nomenclature of Prokaryotes (Oren et al., 2015; Parker et al., 2015; see also Methods). For lower level ranks, notably genus, existing names were often retained with alphabetical suffixing to resolve polyphyly in the bac120 tree (e.g., Bacillus_A, Bacillus_B, etc). Only the group 130 containing type material (if known) kept the original unsuffixed name to indicate validity of name assignment. This serves two purposes, it preserves continuity in the literature, and avoids the necessity to propose dozens of new names for highly polyphyletic groups, although ultimately we suggest that this should be done. A total of 436 genera, 152 families and 67 orders were identified as polyphyletic in the tree highlighting important deficiencies in the current

135 taxonomy (Supp. Table 3). The genus Clostridium was the most polyphyletic, representing 121 genera spanning 29 families, followed by Bacillus (81 genera across 25 families), and Eubacterium (30 genera across 8 families). Note, however, that these numbers were also influenced by rank normalization in some cases (see below).

\section{Rank normalization}

There is currently no accepted standardized approach for assigning taxa to higher ranks (i.e., genus to phylum), although $16 \mathrm{~S}$ rRNA sequence identity and amino acid identity (AAI) thresholds have been proposed (Hugenholtz et al., 1998; Yarza et al., 2014; Konstantinidis \& Tiedje, 2005). Assignment of ranks within the NCBI taxonomy is highly variable under both

145 these measures as they have been proposed relatively recently and have not been widely adopted (Yarza et al., 2014; Hugenholtz et al., 2016). We normalized the assignment of higher taxonomic ranks using relative evolutionary divergence (RED) calculated from the bac120 tree, which is 
conceptually similar to the approach used by Wu et al. (2013b). Our approach provides an operational approximation of relative time with extant taxa existing in the present $(\mathrm{RED}=1)$, the last common ancestor occurring at a fixed time in the past $(\mathrm{RED}=0)$, and internal nodes being linearly interpolated between these values according to lineage-specific rates of evolution (Fig. 1; see Methods). RED intervals for normalizing taxonomic ranks were defined as the median RED value for taxa at each rank \pm 0.1 (Fig. 1). This represents a compromise between strict normalization and the desire to preserve existing group names on well-supported interior nodes.

155 Visualization of the NCBI taxonomy according to RED highlights a substantial number of overor under-classified taxa (Fig. 2a). To correct these inconsistencies, taxa falling outside of their RED intervals were either reassigned to a new rank (with appropriate nomenclatural changes) or to a new node in the tree (Fig. 2b).

160 In contrast to $16 \mathrm{~S}$ rRNA sequence identity or AAI thresholds, RED normalization accounts for the phylogenetic relationships between taxa and variable rates of evolution. For example, members of the rapidly evolving genus Mycoplasma (Maniloff, 2002) are sufficiently diverged to represent two phyla using a 16S rRNA gene sequence identity threshold of 75\% (Yarza et al., 2014). However, vertebrate-associated Mycoplasma and Ureaplasma diverged from their

165 arthropod-associated sister families only 400 million years ago (Maniloff, 2002), approximately consistent with the emergence of vertebrates (Kumar et al., 2017). This evolutionary event occurred much later than the primary diversification of bacterial phyla, which is estimated to have occurred between two and three billion years ago (Marin et al., 2016). The relatively recent emergence of Mycoplasma is more consistent with their RED-normalized ranking into a single

170 order within the Firmicutes, than the two phyla that a $16 \mathrm{~S}$ rRNA sequence identity of $75 \%$ would indicate.

\section{Validation of the GTDB taxonomy}

The robustness of the approach used to generate the GTDB taxonomy was evaluated by varying marker sets, taxa, or evolutionary models. We first considered a tree inferred from a syntenic

175 block of 16 ribosomal proteins (rp1; Brown et al., 2015; Hug et al., 2016; Parks et al., 2017) and determined the optimal position of each GTDB taxon within this tree (see Methods). On average, $94.7 \%$ of GTDB taxa at each rank were monophyletic or operationally monophyletic (defined as 
having an F-measure $\geq 0.95$ ) within the rp1 tree, with the least being $92.7 \%$ at the class level and the most being $96.5 \%$ at the order level (Fig. 3; Supp. Fig. 1a; Supp. Table 1). Taxa that were not monophyletic within the rp1 tree were most often due to the incongruent placement of a small number of genomes, resulting in direct conflict with the GTDB taxonomy or simply unresolved as groups in the rp1 tree (see Methods). Less than $0.5 \%$ of genomes had a conflicting taxonomic assignment at any rank, and $<1.5 \%$ had an unresolved taxonomic assignment at any rank with the exception of order-level assignments which were unresolved for $4.0 \%$ of genomes

185 (Supp. Fig. 1b; Supp. Table 4). We also observed that taxa at the same taxonomic rank had similar RED values indicating that rank normalization was largely preserved in the rp1 tree (Fig. 3; Supp. Fig. 1c). Performing the same analysis on a $16 \mathrm{~S}$ rRNA gene tree resulted in $78.1 \%$ (species) to $90.8 \%$ (class) of GTDB taxa being recovered as monophyletic or operationally monophyletic (Supp. Fig. 2a). Incongruent taxonomic assignments in the 16S rRNA tree were 190 largely the result of unresolved taxa with $<1.1 \%$ of genomes having conflicting assignments at any rank (Supp. Fig. 2b; Supp. Table 5). Taxa at the same rank had similar RED values in the $16 \mathrm{~S}$ rRNA gene tree, though the spread of values was greater than observed on the bac120 or rp1 trees (Fig. 3; Supp. Fig. 2c).

195 For comparison, we evaluated the congruence of the NCBI taxonomy with the topologies of the bac120, rp1 and 16S rRNA trees. All three trees had numerous discrepancies with the NCBI taxonomy both in terms of polyphyly and over- and under-classified taxa (Figs. 2 and 3). On average, $26.3 \%, 26.1 \%$ and $26.6 \%$ of NCBI taxa were classified as polyphyletic on the bac120, rp1 and 16S rRNA trees, respectively, with variable distributions across the ranks (Supp. Figs. 3

200 to 5). Curation of the GTDB on the bac120 tree ensures all taxa are monophyletic on this tree and consequently the GTDB taxa show markedly less polyphyly on the rp1 (5.3\%) and 16S rRNA (14.6\%) trees than NCBI taxa. In total $64.1 \%, 60.2 \%$ and $59.5 \%$ of RefSeq/GenBank genomes had an NCBI taxonomy congruent with the bac120, rp1 and 16S rRNA trees, while $89.8 \%$ and $76.1 \%$ of these genomes had GTDB assignments in agreement with the rp1 and 16S 205 rRNA trees. Furthermore, the NCBI taxonomy had a poorer RED fit than the GTDB taxonomy at all ranks in these three trees (Fig. 3). 
The stability of the GTDB taxonomy on trees inferred under marker set and taxon subsampling was also evaluated. Subsampling of the 120 bacterial marker genes was performed 100 times 210 with 60 of the markers randomly selected for each replicate. Notably, 96.7\% of GTDB taxa were classified as monophyletic in $\geq 90 \%$ of the replicate trees and only 10 taxa $(0.11 \%)$ were classified as polyphyletic in $\geq 50 \%$ of replicates (Supp. Table 1). Given the lower phylogenetic resolution of individual genes (Gadagkar et al., 2005; Lang et al., 2013), results from individual gene trees were also remarkably robust with $86.1 \%$ of GTDB taxa being monophyletic in $\geq 50 \%$

215 of trees (Supp. Table 1). Taxon resampling with one genome per genus was performed 100 times with representative genomes being randomly selected each replicate. Across the 1,430 taxa with two or more genera, $97.5 \%$ were recovered as monophyletic in $\geq 90 \%$ of the taxonresampled trees and only four taxa (f_F082, f__Hyphomonadaceae, c_Bacilli_A, $\mathrm{p} \_$_Desulfobacteraeota) were classified as polyphyletic in $\geq 50 \%$ of replicates (Supp. Table 1).

220 The proposed taxonomy was also robust to model selection with only two GTDB taxa being operationally monophyletic ( polyphyletic (c_Bacilli_A; o__Ryanbacterales; s__Blastomonas delafieldii) under a tree inferred with the LG protein substitution model (Le \& Gascuel, 2008) instead of the WAG model (Whelan \& Goldman, 2001; Supp. Table 1).

\section{Comparison of the GTDB and NCBI taxonomies}

Overall, $73 \%$ of the 84,634 genomes with an NCBI taxonomy had one or more changes to their classification above the rank of species (Fig. 4a). These included both reclassification of taxa and filling in missing rank name information ( $3 \%$ of genus to phylum names are currently undefined across the 84,634 genomes with an NCBI taxonomy). On average, $22 \%$ of names were

230 changed per rank, the least being $7 \%$ at the phylum level, and the most being $50 \%$ at the order level (Fig. 4a). A total of 199 NCBI names above the rank of species were 'retired' from the GTDB taxonomy mostly as a result of RED normalization (Supp. Table 6). A detailed listing of changes overviewed in Fig. 4a can be found in Supp. Table 3. Only 18\% of GTDB taxa above the rank of species were validly published and approved, a further $19 \%$ proposed but not

235 validated (many of these lack type material), and 63\% were non-standard placeholder names (Fig. 4b) indicating the scope of the task remaining to produce a fully standardized and validated taxonomy. This task will be greatly facilitated by recent proposals to use genome sequences as 
type material for as-yet-uncultured lineages, which in principle allows validation of names (Whitman 2016; Konstantinidis et al., 2017).

\section{Genus- and species-level classifications}

Genera and species comprise $84 \%$ of the 16,924 defined taxon names in the bac120 tree. Misclassified species in the public repositories are a particular area of concern to researchers as they can introduce noise into a variety of analyses, including strain typing (Comas et al., 2009),

245 biogeographic distributions of species (Martiny et al., 2006) and pangenome analyses (Trost et al., 2010). Moreover, classification errors can propagate over time as incorrectly labeled genomes are used as reference material to identify novel sequences. A small number of microbial genera have been rigorously examined for this problem and taxonomic corrections proposed, including Aeromonas (Beaz-Hidalgo et al., 2015) and Fusobacterium (Kook et al., 2017). We

250 compared the results of these analyses to the GTDB taxonomy as a means of providing independent verification of our results. Based on multilocus sequence analysis and average nucleotide identity (ANI) comparisons, Beaz-Hidalgo et al. (2015) proposed that nine Aeromonas dhakensis genomes are incorrectly classified as A. hydrophila. All nine of these strains were reclassified as A. dhakensis in the bac120 tree, and an additional four genomes not

255 included in the Beaz-Hidalgo study were also reclassified as A. dhakensis (Supp. Table 7). Kook et al. (2017) recently recommended the reclassification of Fusobacterium nucleatum subspecies animalis, nucleatum, polymorphum, and vincentii as separate species based on ANI and genome distance metrics. Rank normalization of the GTDB taxonomy using RED values largely reproduced this finding without prior knowledge of the authors' work (Supp. Table 7).

260 Reclassification of species according to the bac120 tree is also consistent with recent efforts to objectively define bacterial species based on barriers to homologous recombination estimated against the core genome of each species (Bobay \& Ochman, 2017). In that study, 23 of 91 bacterial species were proposed to contain one or more members not belonging to their respective species ('excluded taxa'). We found that almost all comparable instances of excluded 265 taxa were due to misclassification in the NCBI taxonomy (Supp. Table 7). These results suggest that the bac120 tree topology and RED estimates of species-level groups based on $\sim 4 \%$ of the genome (120 conserved markers) are consistent with alternative analytical approaches using larger fractions of the genome. 
270 The genus Clostridium is widely acknowledged to be polyphyletic and efforts have been made to rectify this problem, including a global attempt to reclassify the genus using a combination of phylogenetic markers (Yutin \& Galperin, 2013). These authors proposed the reclassification of 78 Clostridium species, and nine other species, into six novel genera (Yutin \& Galperin, 2013; Galperin et al., 2016). Of these, we could confirm that Erysipelatoclostridium (with the 275 exception of C. innocuum str. 2959), Gottschalkia and Tyzzerella (excepting C. nexile CAG:348) represent monophyletic genus-level groups. The remaining three genera proposed by Yutin \& Galperin (2013) represent multiple genera in the GTDB taxonomy including validly published genera (Supp. Table 8). This is consistent with several recent analyses of individual taxa in these groups (Yarza et al., 2008; Lawson et al., 2016; Sakamoto et al., 2017). The GTDB

280 taxonomy is also largely in agreement at the genus level with a recent global genome-based classification of the Bacteroidetes (Hahnke et al., 2016). Of the 122 genera addressed in that study, six were found to be in need of reclassification; Chryseobacterium, Epilithonimonas, Aequorivita, Vitellibacter, Flexibacter, and Pedobacter. All six were similarly identified as polyphyletic in the GTDB taxonomy and reclassified accordingly. These findings demonstrate 285 that our methods are broadly consistent with rigorous, independent analyses of problematic genera and species.

\section{Higher-level classifications}

A number of notable taxonomic changes at higher ranks are proposed for well-studied groups.

290 For example, the class Betaproteobacteria was reclassified as an order within the class Gammaproteobacteria because it is entirely circumscribed within the latter group, and is closer to the median RED value for an order than a class (Fig. 2a). This change is consistent with the original 16S rRNA gene topology of the Proteobacteria and subsequent trees (Ludwig \& Klenk, 2001; Garrity et al., 2005; McDonald et al., 2012; Quast et al., 2013; Yilmaz et al., 2014),

295 although such a rank change was not proposed in these studies. The Deltaproteobacteria and Epsilonproteobacteria have been removed entirely from the Proteobacteria as this phylum is not consistently recovered as a monophyletic unit, as found in many previous $16 \mathrm{~S}$ rRNA and other marker gene analyses (e.g., Rinke et al, 2013; Yarza et al., 2014; Waite et al., 2017). In the case 
of the Epsilonproteobacteria, this class was combined with the order Desulfurellales

300 (Deltaproteobacteria) to form a new phylum, the Epsilonbacteraeota (Waite et al., 2017).

The Firmicutes also underwent extensive internal reclassification. As a clade, this phylum is typically monophyletic but poorly supported in most trees (Supp. Table 1), and has a RED in the phylum range, albeit to the left of the median for this rank (Fig. 2b). The Firmicutes have 305 therefore been retained as a phylum-level lineage, although future revision of this status may be warranted. The taxa that comprise this phylum have been divided into 34 classes including the mycoplasmas which are currently classified as a separate phylum, the Tenericutes (Brown, 2010), and 14 classes exclusively comprised of MAGs. Incorporation of the Tenericutes within the Firmicutes is consistent with single-gene phylogenies (Wolf et al., 2004; Yarza et al., 2008;

310 McDonald et al., 2012; Quast et al., 2013), and is further supported by recent evidence using multiple molecular markers (Lang et al., 2013; Segata et al., 2013; Hug et al., 2016; Skennerton et al., 2016). Similar to its type genus, the order Clostridiales has been extensively subdivided (Fig. 5a) largely as a consequence of an anomalous RED for this rank (Fig. 2a).

315 Based on robust monophyly, rank normalization and naming priority in the literature, the phylum Bacteroidetes is proposed to encompass the Chlorobi (Garrity et al., 2001) and Ignavibacteriae (Podosokorskaya et al., 2013) as class-level lineages. Concomitantly, several former classes of Bacteroidetes have been amalgamated into the class Bacteroidia as order-level lineages including the Chitinophagales, Cytophagales, Flavobacteriales and Sphingobacteriales (Fig.

320 5b). These proposed changes are in contrast to recent reclassifications, in which Bacteroidetes is divided into three major lineages by promoting the families Rhodothermaceae and Balneolaceae to phyla (Fig. 2a; Hahnke et al., 2016; Munoz et al., 2016). In the GTDB taxonomy, these are retained as families within their own orders in the class Rhodothermia based on their RED values (Fig. 2b). The higher-level taxonomy of the phylum Actinobacteria is largely unchanged. The 325 five classes Actinobacteria, Acidimicrobiia, Coriobacteriia, Thermoleophilia, and Rubrobacteria are retained with the sole change at the class-level being the downgrading of the Nitriliruptoria (Ludwig et al., 2012) to an order within the class Actinobacteria based on rank normalization. Changes to other major lineages are summarized in Supp. Table 3. 


\section{Calibration of uncultured microbial diversity}

Having normalized the taxonomy on existing isolate-based classifications, we were able to calibrate the taxonomic ranks of uncultured lineages. Candidate phylum KSB3 was initially proposed based on comparative analysis of environmental 16S rRNA gene sequences (Tanner et al., 2000; Yamada et al., 2007), and more recently two near-complete MAGs belonging to this

335 phylum were reconstructed from a bulking sludge metagenome, for which the names Candidatus Moduliflexus flocculans and Ca. Vecturathrix granuli were proposed (Sekiguchi et al., 2015). These genomes were further classified into separate families, orders and classes within the phylum, however, by rank normalization they only represent separate genera belonging to a single family. The group still retains a phylum-level status as it is not reproducibly affiliated with

340 other bacterial lineages (Hugenholtz et al., 1998); however, we propose that the phylum (Modulibacteria) is currently genomically represented by a single class (Moduliflexia), single order (Moduliflexales) and single family (Moduliflexaceae; Fig. 2b).

As part of a single cell genomics study, the superphylum Patescibacteria was proposed to 345 encompass the candidate phyla Parcubacteria (OD1), Microgenomates (OP11), and Gracilibacteria (GN02) (Rinke et al., 2013). These candidate phyla were further subsumed within the Candidate Phyla Radiation (CPR) based on the addition of 797 MAGs (Brown et al., 2015). Currently there are at least 65 candidate phyla proposed to belong to the CPR (Brown et al., 2015; Anantharaman et al., 2016), with the justification of individual phyla based primarily on a

$35016 \mathrm{~S}$ rRNA sequence identity threshold of 75\% (Yarza et al., 2014). The CPR is consistently recovered as a monophyletic group using concatenated protein markers in this and previous studies (Brown et al., 2015; Hug et al., 2016; Parks et al., 2017). However, rank normalization suggests that the CPR should be reclassified as a single phylum for which we have reimplemented the name Patescibacteria (Fig. 2), with concomitant subordinate changes.

\section{Outlook}

The GTDB taxonomy aims to provide an objective, phylogenetically consistent classification of bacterial species, and we have shown that it is largely congruent with the topology and substitution rates inferred under different gene sets and models of evolution. While we have preserved existing taxonomic classifications where possible, a substantial number of 
360 modifications were required in order to resolve polyphyletic groups and to normalize taxa at each rank based on our operational approximation of relative time of divergence. The GTDB taxonomy covers 94,759 bacterial genomes, but we expect the number of available reference genomes to expand rapidly and to quickly encompass new lineages (Anantharaman et al., 2016; Parks et al., 2017). In anticipation of this expansion, we plan to curate the taxonomy biannually

365 in order to incorporate new genomes and proposed taxonomic groups while retaining a phylogenetically consistent classification. Subsampling of the bac120 dataset suggests that subsets of these marker genes could be used in the future to produce reliable phylogenies that better scale with the projected increase in the reference genome database (Hugenholtz et al., 2016). Some incongruencies between genome trees inferred for each biannual update are

370 expected to impact the GTDB taxonomy as has already been observed for well-established groups such as the Firmicutes, which may require reclassification in subsequent iterations. Ideally, such regions of instability should be addressed individually with more in-depth analyses to establish the most suitable classification, as for example, was done recently with the class Epsilonproteobacteria (Waite et al., 2017). The GTDB taxonomy is available through the

375 Genome Taxonomy Database website (http://gtdb.ecogenomic.org) and we are facilitating its incorporation into other public bioinformatic resources. We are also developing a standalone tool to enable researchers to classify their own genomes according to the GTDB taxonomy and its classification criteria. The methodology described here is applicable to any taxonomically annotated phylogenetic tree and we are in the process of expanding the taxonomy to Archaea and

380 double-stranded DNA viruses. We anticipate that the availability of an up-to-date normalized genome-based classification will greatly facilitate analysis of microbial genome data and communication of scientific results.

\section{Methods}

\section{Genome dataset}

385 A dataset of 87,106 bacterial genomes was obtained from RefSeq/GenBank release 80 (Haft et al., 2017) on January 17, 2017. An additional 11,603 MAGs obtained from Sequence Read Archive metagenomes (Leinonen et al., 2011) were added to this dataset to improve coverage of uncultured lineages, most of which have been reported previously (Parks et al., 2017). These genomes were dereplicated as described in Parks et al. (2017) with the exception that 
390 dereplication was based on ANI values estimated using Mash distances (Ondov et al., 2016) instead of pairwise AAI values calculated from the bac120 alignment. Specifically, a genome was assigned to a representative genome if one of the following criteria was met: i) the Mash distance was $\leq 0.1$ ( $\sim$ ANI of $90 \%$ ) and the genomes had the same species assignment at NCBI, ii) the Mash distance was $\leq 0.05$ ( $\sim$ ANI of 95\%) and the genomes had the same species assignment

395 in the previous iteration of the GTDB taxonomy (release 78; http://gtdb.ecogenomic.org/ downloads), or iii) the Mash distance was $\leq 0.035$ ( $\sim$ ANI of $96.5 \%$ ) and the query genomes had no species assignment at NCBI or in the previous iteration of the GTDB taxonomy. Following dereplication, genomes were excluded that had i) amino acids in $<50 \%$ of the columns within the bac120 alignment and/or ii) an estimated quality $<50$, defined as completeness $-5 \times$

400 contamination and calculated using the default lineage-specific marker gene sets of CheckM (Parks et al., 2015).

\section{Metadata}

The NCBI taxonomy associated with the reference genomes was obtained from the NCBI 405 Taxonomy FTP site on January 17, 2017. This taxonomy was standardized to seven ranks (domain to species) by removing non-standard ranks and identifying missing standard ranks with rank prefixes. Standard ranks were also prefixed with rank identifiers as previously described (McDonald et al., 2012). For example, the full NCBI lineage for 'Nostoc azollae' 0708 (GCF_000196515.1) at the time of download was "cellular organisms (no rank); Bacteria 410 (superkingdom); Terrabacteria group (no rank); Cyanobacteria/Melainabacteria group (no rank); Cyanobacteria (phylum); Nostocales (order); Nostocaceae (family); Trichormus (genus); Trichormus azollae (species); 'Nostoc azollae' 0708 (strain)", which was standardized to "d__Bacteria; p__Cyanobacteria; c__; o__Nostocales; f__Nostocaceae; g_trichormus; s_trichormus azollae". Additional metadata from NCBI such as "Isolate", "Assembly level" 415 and "Genome representation" were also parsed from the assembly reports of all bacterial genomes (http://gtdb.ecogenomic.org/downloads) to provide information for manual tree curation. To complement the NCBI taxonomy and metadata, 16S rRNA gene sequences were identified from all NCBI genomes and MAGs using HMMER v3.1b1 (Eddy, 2011) and the most similar sequence within the Greengenes 2013 (McDonald et al., 2012) and SILVA v123.1

420 (Yilmaz et al., 2014) databases identified using BLASTN v2.2.30+ (Camacho et al., 2009). 
Inference and annotation of the bac120 tree

A phylogenetic tree spanning the dereplicated genomes was inferred from the concatenation of 120 ubiquitous, single-copy marker genes (bac120 marker set) identified within the Pfam v27

425 (Finn et al., 2014) and TIGRFAMs v15.0 (Haft et al., 2003) databases, which had previously been evaluated to be phylogenetically informative (Parks et al., 2017). Gene calling was performed with Prodigal v2.6.3 (Hyatt et al., 2010) and the 120 marker proteins identified and aligned using HMMER v3.1b1. The resulting multiple sequence alignment was trimmed by removal of columns represented by $<50 \%$ of taxa and/or with an AA consensus $<25 \%$. In

430 addition, genomes with amino acids in $<50 \%$ of columns were removed before phylogenetic inference. The bac120 reference tree was inferred using FastTree v2.1.7 (Price et al., 2009) under the WAG model of protein evolution (Whelan \& Goldman, 2001) with gamma-distributed rate heterogeneity (+GAMMA; Yang, 1994). Branch support was estimated by performing 100 nonparametric bootstrap replicates. Group names based on the standardized NCBI genome 435 taxonomy were added to interior nodes of the bac120 tree using tax 2 tree (McDonald et al., 2012).

\section{Calculating relative evolutionary divergence and thresholds for taxonomic ranks}

Relative evolutionary divergence (RED) values were calculated from the annotated bac120 tree

440 using PhyloRank (v0.0.27; https://github.com/dparks1134/PhyloRank). PhyloRank performs a preorder tree traversal with the RED of the root defined to be zero and the RED of node $n$ defined as $p+(d / u)(1-p)$, where $p$ is the RED of its parent, $d$ is the branch length to its parent, and $u$ is the average branch length from the parent node to all extant taxa descendant from $n$. As the RED of taxa is influenced by root placement and the rooting of the bacterial tree remains

445 controversial (Williams et al., 2015), we took an operational approach and rooted trees at the midpoint of all branches leading to phyla with $\geq 2$ classes. The RED of a taxon was then taken as the median RED over all tree rootings, excluding the tree in which the taxon was part of the outgroup. Median RED values for each taxonomic rank were determined from taxa with $\geq 2$ immediately subordinate taxa (e.g., phyla with $\geq 2$ defined classes) and the RED rank intervals 450 used to guide the GTDB taxonomy defined as \pm 0.1 from these median RED values. 


\section{Tree-based taxonomic curation}

The annotated bac120 tree was manually curated in ARB (Ludwig et al., 2004) to i) resolve polyphyletic groups, ii) correct taxa falling outside of their RED distribution and iii) add 16S

455 rRNA-based group names to uncultured lineages. Branch lengths in the bac120 tree were replaced with their corresponding RED values to produce a 'scaled' tree as a visual aid in the rank normalization process (Fig. 1c). Polyphyletic groups were identified as part of the initial annotation of group names with numerical suffixes generated by tax 2 tree. Groups containing type material according to the List of Prokaryotic Names with Standing in Nomenclature (LPSN;

460 Euzeby, 1997) kept the original unsuffixed name to indicate validity of name assignment, and other groups were renamed according to a set of nomenclatural rules (see below). Outlier group names $( \pm 0.1$ from the median RED values) were moved into their rank distributions in one of two ways; the name was moved to another interior node in the bac120 tree, or the name was left on the original interior node but reclassified to a different rank. 16S rRNA taxonomy-based

465 names were assigned to clades in the bac120 tree if one or more genomes spanning the clade had $\geq 95 \%$ identity over $\geq 500$ bp to a reference $16 \mathrm{~S}$ rRNA sequence with a given name. Robust interior nodes (bootstrap support $>90 \%$ ) were given preference for name assignments.

\section{Generation of final GTDB taxonomy}

470 The GTDB taxonomy was extracted from the curated bac120 tree (Newick input format) by concatenating group names from the relevant interior nodes for each genome, and exporting them as a flat text file for validation. Validation included checks for correct number and order of ranks, presence of multiple-parents (polyphyly), orthographic and semantic errors, and consistency of order (-ales) and family (-aceae) rank suffixes. As names can only be applied to

475 groups of $\geq 2$ taxa in ARB, 'singleton' genomes often have incomplete taxonomic lineages in the exported flat text file. These were auto-completed to at least the level of genus based on the nomenclatural rules outlined below. Consistency of filled ranks between releases was tracked using additional scripts and the completed taxonomy validated once more.

\section{Nomenclatural rules for standard names}

Nomenclatural changes of validly published names were made according to the International Code of Nomenclature of Prokaryotes (ICNP; Parker et al., 2015). In the event of the 
nomenclature type been excluded from or not present in the group, a new type was designated based on priority in the literature and provisional higher rank names were established with the 485 addition of corresponding rank suffixes to the stem of the generic name. This includes the recently proposed standard suffix -aeota for the rank of phylum (Oren et al., 2015). Priority was established for all other taxa names, namely those without standing in nomenclature and Candidatus taxa, based on the earliest published taxon in the group, and ranks with missing annotations derived their name from the corresponding generic name of the earliest named taxon.

490 The term Candidatus was removed from GTDB taxon names to standardize the taxonomy but is easily tracked via the NCBI Organism Name in the genome metadata (http://gtdb.ecogenomic.org). In cases where new names were not proposed to resolve polyphyly, notably for the rank of genus, alphabetical suffixes were added to the standard name (e.g., Bacillus_A, Bacillus_B, etc). Species-level groups with non-standard or ambiguous names were 495 designated as 'genus name' sp1, 'genus name' sp2, etc. The official naming hierarchy from lower to higher ranks was followed, with the exception of some provisional phylum names lacking named species (notably CPR phyla), which were retained after rank normalization with appropriate rank suffix changes, e.g. o__Levybacterales from Candidatus Levybacteria (Brown et al., 2015).

Nomenclatural rules for non-standard placeholder names

Non-standard placeholder names were given to groups lacking standardly named representatives. Several sources were used to derive non-standard names; i) 16S rRNA environmental clone names grafted onto the bac120 tree (see above; Supp. Table 2), ii) isolate strain names, e.g. 505 g__Mor1 from the genome "Acidobacteria bacterium Mor1” (GCA_001664505.1), iii) MAG names, e.g. g_UBA4820 from “SRA genome UBA4820” (GCA_002402325.1), and iv) genome assembly identifiers for groups exclusively comprising complex symbiont names, e.g. g_GCF001602625 for "Sodalis-like endosymbiont of Proechinophthirus fluctus" (GCF_001602625.1). Non-standard names longer than 15 characters were trimmed for brevity and to minimize 510 spelling errors, e.g. g_2-02-FULL-67-57 from the name "Acidobacteria bacterium RIFCSPHIGHO2_02_FULL_67_57” (GCA_001766975.1). In the rare event of identical placeholder names for two or more phylogenetically distinct groups resulting from automated name trimming, or rank filling, we appended hyphenated alphabetical suffixes (-A, -B etc.) to 
distinguish them. As with standard binomial names, species-level groups were defined as 'genus

515 name' sp1, 'genus name' sp2 etc. Where necessary, non-standard names were propagated to higher ranks differentiated only by rank prefix, e.g. d__Bacteria; p_Acidobacteria; c__UBA4820; o__UBA4820; f_UBA4820; g_UBA4820.

\section{Inference of trees used to validate the GTDB taxonomy}

520 The stability of the GTDB taxonomy was evaluated using trees inferred in a manner analogous to that described for the bac120 tree. Briefly, proteins were called with Prodigal, marker genes identified and aligned using HMMER with Pfam and TIGRfam HMMs, multiple sequence alignments trimmed based on consistency and ubiquity, genomes with poor representation in the alignment removed before phylogenetic inference, and trees inferred with FastTree under the

525 WAG+GAMMA models unless otherwise specified.

Alternative Marker Sets. A ribosomal protein tree (rp1) was inferred from the concatenation of 16 ribosomal proteins (Brown et al., 2015; Parks et al., 2017) and consisted of 1,949 aligned columns after trimming 101 columns represented by $<50 \%$ of taxa and 11 columns with an 530 amino acid consensus <25\%. The rp1 tree spanned 21,444 genomes after removing 1,967 genomes with amino acids in $<50 \%$ of the filtered columns. Subsampled marker trees were inferred by randomly selecting 60 genes from the bac120 marker set. A total of 100 replicate trees were generated in this fashion in order to assess the impact of using different subsets of the bac120 marker set. The filtered multiple sequence alignments ranged in length from 15,010 to

53520,061 amino acids and all trees spanned 21,943 genomes as no additional genome filtering was performed. Individual gene trees were also inferred for each of the genes comprising the bac120 marker set. The filtered alignments for these trees ranged in length from 43 to 1,069 amino acids and spanned 16,932 to 21,050 genomes.

540 Alternative Taxa. Trees were inferred from sets of taxa subsampled to one genome per genus in the GTDB taxonomy. Representative genomes for each genus were randomly selected from the 21,943 dereplicated genomes comprising the bac120 tree. The alignments used for the full bac120 tree were used to infer 100 taxa-subsampled trees. 
545 Alternative Models. A bac120 tree under the LG (Le and Gascuel, 2008) model was inferred using FastTree v2.1.7 compiled for double precision in order to avoid numerically unstable issues. This tree was inferred using the same multiple sequence alignment as used for the bac120 tree under the WAG+GAMMA models, and spans the same set of genomes.

550 Inference of $16 S$ rRNA gene tree. A 16S rRNA gene tree was inferred from genes $>1,200 \mathrm{bp}$ identified within the 21,943 dereplicated and quality controlled genomes. The 16S rRNA genes were identified using HMMER and domain-specific SSU/LSU HMM models as implemented in the 'ssu-finder' method of CheckM, with the longest gene being retained for genomes with multiple copies of the $16 \mathrm{~S}$ rRNA gene. The 12,712 identified $16 \mathrm{~S}$ rRNA genes were filtered to 555 remove sequences potentially representing contamination using a reciprocal BLAST protocol. Genes were searched against each other using BLASTN v2.2.30+ and a gene was removed from consideration if its closest match belonged to a genome classified in a different taxonomic order as defined by the GTDB, the gene had an alignment length $\geq 800 \mathrm{bp}$, and the gene had a percent identity $\geq 82 \%$. The percent identity criterion was based on the thresholds proposed by Yarza et

560 al. (2014). This results in 277 sequences being removed from consideration (Supp. Table 9). The remaining 12,435 16S rRNA genes were aligned with ssu-align v0.1 (Nawrocki et al., 2009) and trailing or leading columns represented by $\leq 70 \%$ of taxa trimmed which resulted in an alignment of 1,409 bp. The gene tree were inferred with FastTree v2.1.7 under the GTR (Tavaré, 1986) and GAMMA models.

Assessing stability of the NCBI and GTDB taxonomy

The congruency of the NCBI and GTDB taxonomies on different trees was assessed by placing each taxon on the node with the highest resulting F-measure. The F-measure is the harmonic mean of precision and recall, and has been previously proposed for decorating trees with a donor

570 taxonomy (McDonald et al., 2012). Taxonomic stability was assessed as both a percentage of taxonomic groups identified as being monophyletic, operationally monophyletic, or polyphyletic within a tree and the percentage of genomes in the tree with identical, unresolved, or conflicting taxonomic assignments relative to the NCBI or GTDB taxonomies. Since a few incongruent genomes (resulting from phylogenetic incongruence, phylogenetic instability, chimeric artifacts, 575 or erroneous NCBI taxonomic assignments) are sufficient to cause a large number of 
polyphyletic taxa, we classified taxa with an F-measure $\geq 0.95$ as operationally monophyletic. Results were restricted to taxonomic groups containing two or more genomes as taxa represented by a single genome are guaranteed to be monophyletic in a tree. Genomes in a tree with incongruent taxonomic assignments were classified as either i) conflicting if the genome was assigned to a different taxon, or ii) unresolved if the taxon had no taxonomic label at a rank or multiple taxonomic labels at a rank, one of which was the expected label (e.g., a polyphyletic lineage spanning two or more genera, one of which is the expected genus for the genome).

\section{Data availability}

585 Data files for the GTDB taxonomy are available at http://gtdb.ecogenomic.org and include: i) flat file with the GTDB taxonomy defined for 94,759 genomes; ii) bootstrapped bac120 tree in Newick format spanning the 21,943 dereplicated genomes and annotated with the GTDB taxonomy; iii) FASTA files for each marker gene and the trimmed concatenated alignment; iv) metadata for all genomes including NCBI, Silva, and Greengenes taxonomies, completeness and 590 contamination estimates, assembly statistics (e.g., N50), and genomic properties (e.g., GCcontent, genomes size); v) FASTA file of $16 \mathrm{~S}$ rRNA gene sequences identified within the 21,943 dereplicated genomes; and vi) an ARB database containing the bac120 tree. The 3,087 MAGs introduced in this study are available under BioProject PRJNA417962 and on the GTDB website.

\section{Code availability}

RED values were calculated with PhyloRank (https://github.com/dparks1134/PhyloRank) which is freely available under the GNU General Public License v3.0.

\section{Acknowledgements}

600 We thank Pelin Yilmaz for helpful discussions on the proposed genome-based taxonomy, QFAB Bioinformatics for providing computational resources, and members of ACE for beta-testing GTDB. The project is primarily supported by an Australian Research Council Laureate Fellowship (FL150100038) awarded to PH. 
bioRxiv preprint doi: https://doi org/10.1101/256800. this version posted January 31, 2018. The copyright holder for this preprint (which was not certified by peer review) is the author/funder, who has granted bioRxiv a license to display the preprint in perpetuity. It is made available under aCC-BY-NC-ND 4.0 International license.

\section{Supplementary Material}

Methods and Materials

Supp. Tables 1 to 9

Supp. Figures 1 to 5 


\section{Figures}
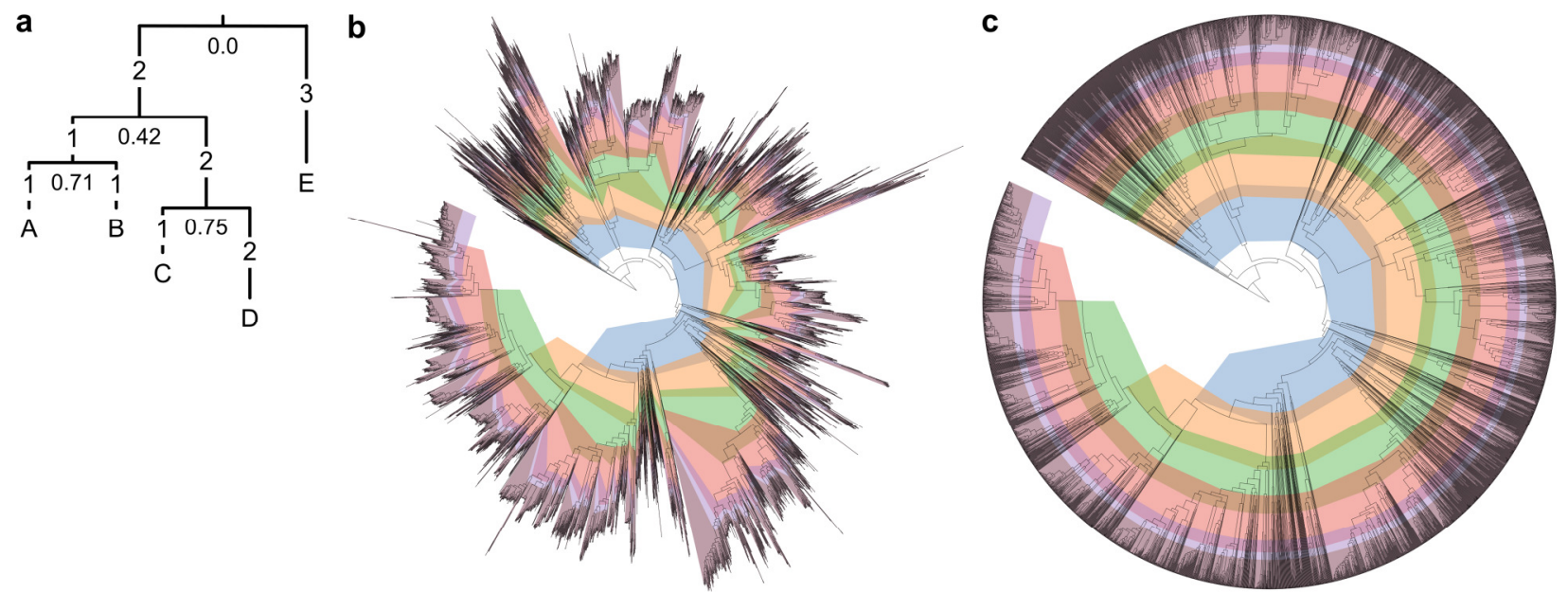

615

OPhylum OClass OOrder OFamily OGenus OSpecies

Figure 1. Rank normalization using relative evolutionary divergence (RED). (a) Example illustrating the calculation of RED. Numbers on branches indicate their length and numbers below each node indicate their RED. The root of the tree is defined to have a RED of zero and leaf nodes have a RED of one. The RED of an internal node $n$ is linearly interpolated from the branch lengths comprising its lineage as defined by $p+(d / u) \times(1-p)$, where $p$ is the RED of its parent, $d$ is the branch length to its parent, and $u$ is the average branch length from the parent node to all extant taxa descendant from $n$. For example, the parent node of leaves $\mathrm{C}$ and $\mathrm{D}$ has a RED value of $0.75(=0.42+(2 / 3.5) \times(1-0.42))$ as its parent has a RED of $p=0.42$, the branch length to the parent node is $d=2$, and the average branch length from the parent node to C and D is $u=(3+4) / 2=3.5$. (b) Bacterial genome tree inferred from 120 concatenated proteins and contoured with the RED interval assigned to each taxonomic rank. Adjacent ranks overlap in some instances as this permits existing group names to be placed on well-supported interior nodes. In order to accommodate visualizing the RED intervals, the initial tree inferred across 21,943 was pruned to 8,142 genomes by retaining two genomes from each species with multiple

630 representatives. The tree is rooted on the phylum Acetothermia for illustrative purposes. RED values used for rank normalization are averaged over multiple plausible rootings (see Methods). (c) The bacterial genome tree with branch lengths scaled by their RED values illustrating that 
rank normalization follows concentric rings that provide an operational approximation of relative time of divergence.
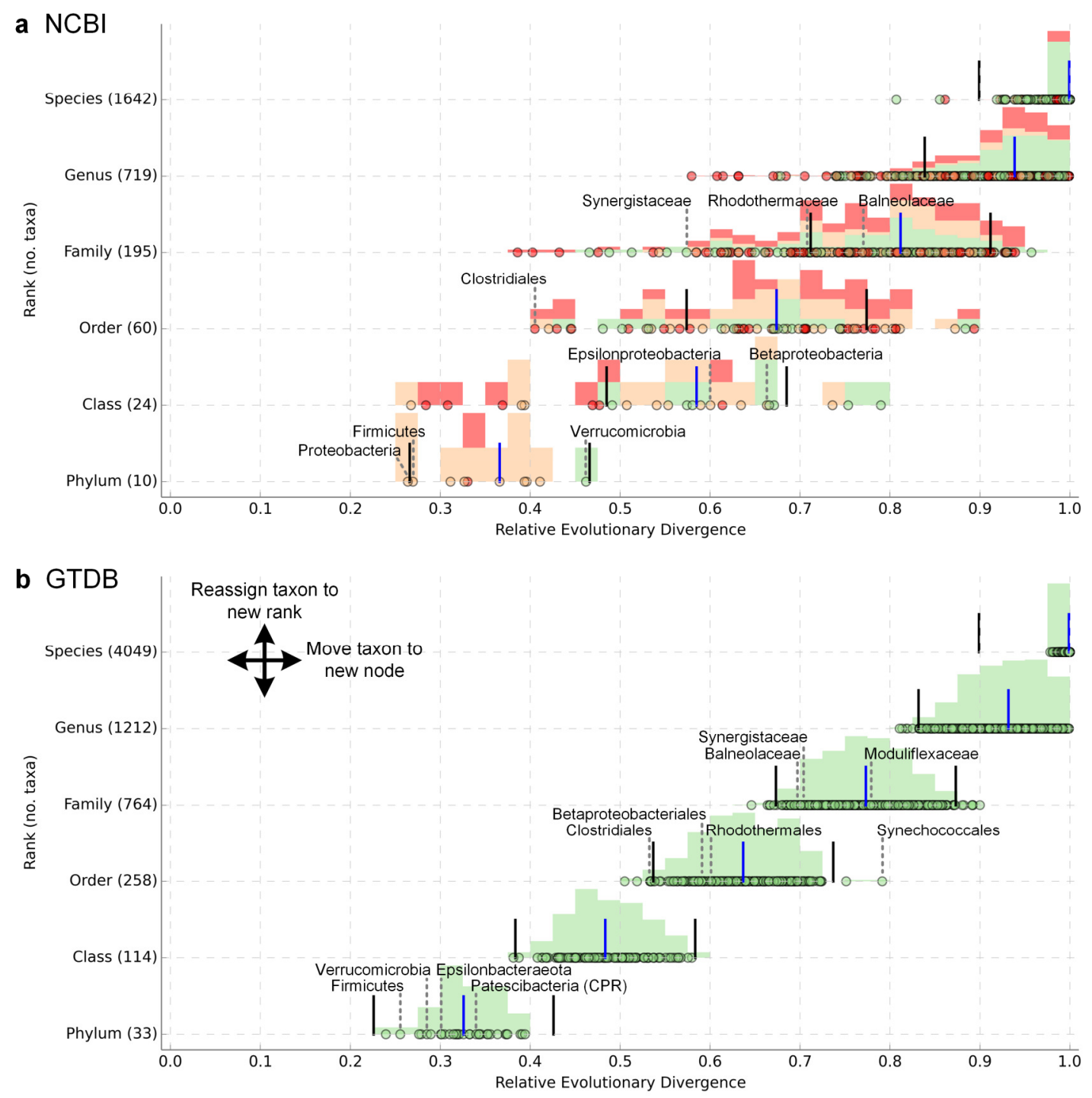

Figure 2. Relative evolutionary divergence of NCBI and GTDB taxa in a genome tree inferred from 120 concatenated proteins. (a, b) RED of taxa defined by the NCBI and GTDB taxonomies, respectively. Each point represents a taxon distributed according to their rank (Yaxis) and colored green, orange, or red to indicate the taxon is monophyletic, operationally monophyletic, or polyphyletic in the genome tree, respectively. A histogram is overlaid on the points to show the relative density of monophyletic, operationally monophyletic, and polyphyletic taxa. The median RED value of each rank is shown by a blue line and the RED interval for each rank shown by black lines. Only monophyletic or operationally monophyletic 
645 taxa were used to calculate the median RED values for each rank. The GTDB aims to resolve taxa that are over- or under-classified based on their RED value by either reassigning them to a new rank (vertical shift in plot) or moving them to a new interior node (horizontal shift in plot). For example, the family Synergistaceae was normalized by reclassifying the family to only encompass the genera Synergistes, Cloacibacillus, Thermanaerovibrio and Aminomonas, rather

650 than the 12 genera circumscribed by this family in the NCBI taxonomy. Only taxa with two or more subordinate taxa are plotted as these taxa have positions in the tree indicative of their rank (e.g., only 33 of the 99 phyla defined by the GTDB contain two or more classes and a phylum with a single class consisting of multiple orders is expected to have a RED value commensurate with the rank of class). The number of taxa plotted at each rank is given in parenthesis along the 655 y-axis. 

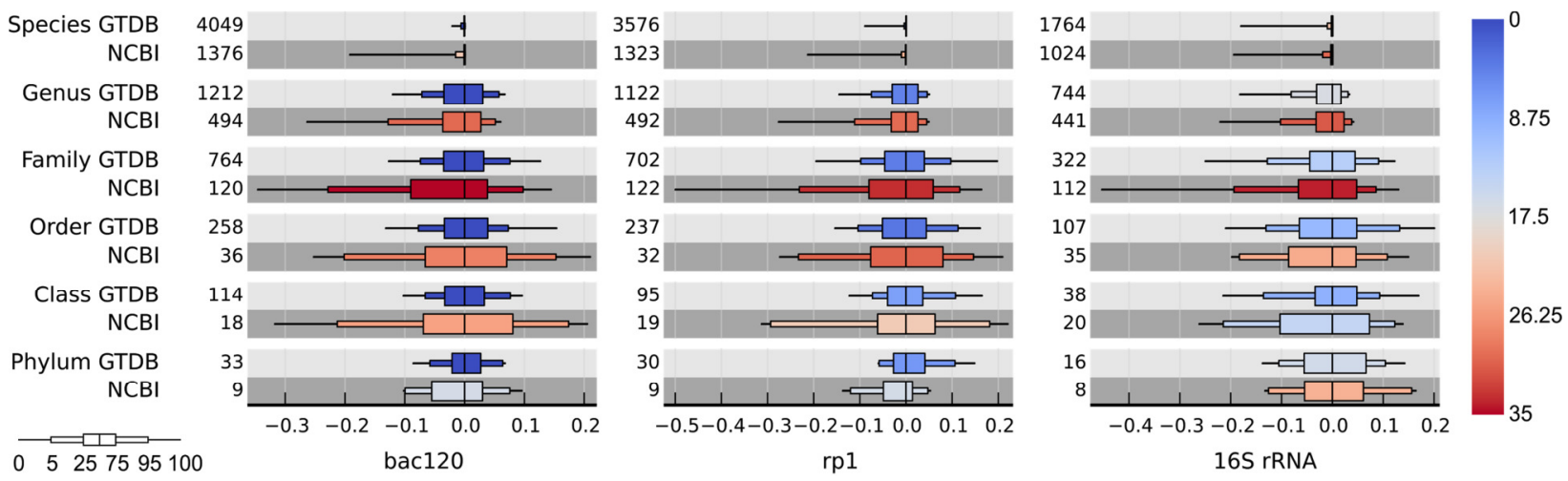

Figure 3. Relative evolutionary divergence (RED) and polyphyly of GTDB and NCBI taxa on trees inferred from 120 bacterial proteins (bac120), 16 ribosomal proteins (rp1), and the $16 S$ rRNA gene. The percentage of taxa classified as polyphyletic in each tree at each rank is indicated by a color gradient from blue to red. RED distributions for taxa at each rank are shown

665 relative to the median RED value of the rank. Results are summarized using box-and-whisker plots indicating the $0^{\text {th }} / 100^{\text {th }}, 5^{\text {th }} / 95^{\text {th }}, 25^{\text {th }} / 75^{\text {th }}$, and $50^{\text {th }}$ percentiles. Distributions were calculated over monophyletic and operationally monophyletic taxa with two or more subordinate taxa as these taxa have positions in the tree indicative of their rank. The number of taxa comprising each distribution is shown next to each box-and-whisker plot. Plots showing the RED values of 670 individual GTDB and NCBI taxa within the bac120, rp1, and 16S rRNA trees are shown in Fig. 2 and Supp. Figs. 1 to 5. 
a

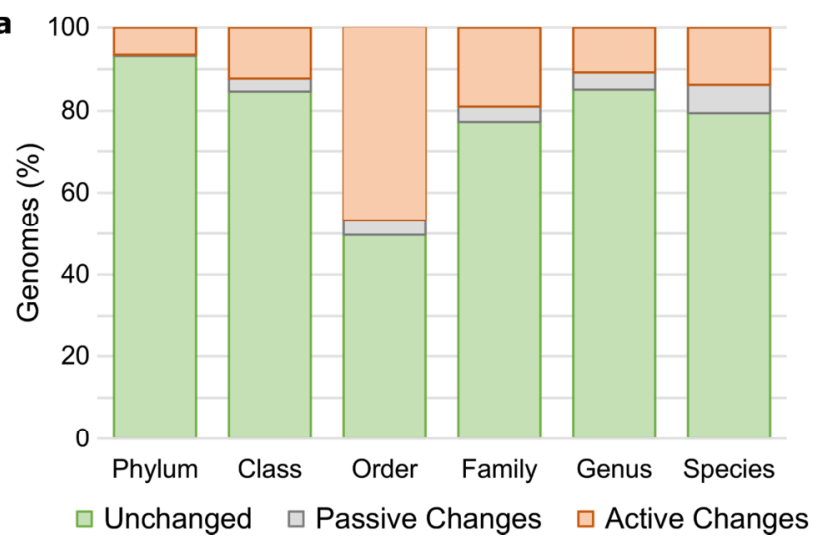

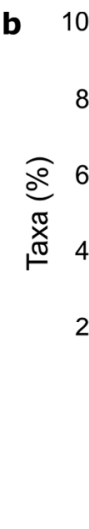

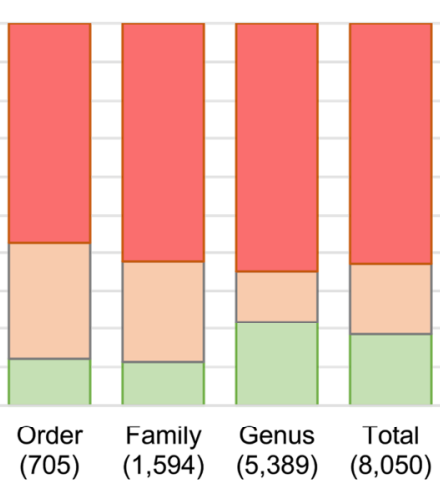

$\square$ Validated $\square$ Proposed $\square$ Placeholder

Figure 4. Comparison of GTDB and NCBI taxonomies and naming status of GTDB taxa.

(a) Comparison of GTDB and NCBI taxonomic assignments across 84,634 bacterial genomes from RefSeq/GenBank release 80. For each rank, a taxon was classified as being unchanged if its name was identical in both taxonomies, passively changed if the GTDB taxonomy provided

685 name information absent in the NCBI taxonomy, or actively changed if the name was different between the two taxonomies. (b) Percentage of GTDB taxa at each rank that are validly published and approved, proposed but not validated, or are non-standard placeholder names. The number of taxa at each rank is shown in parentheses. 


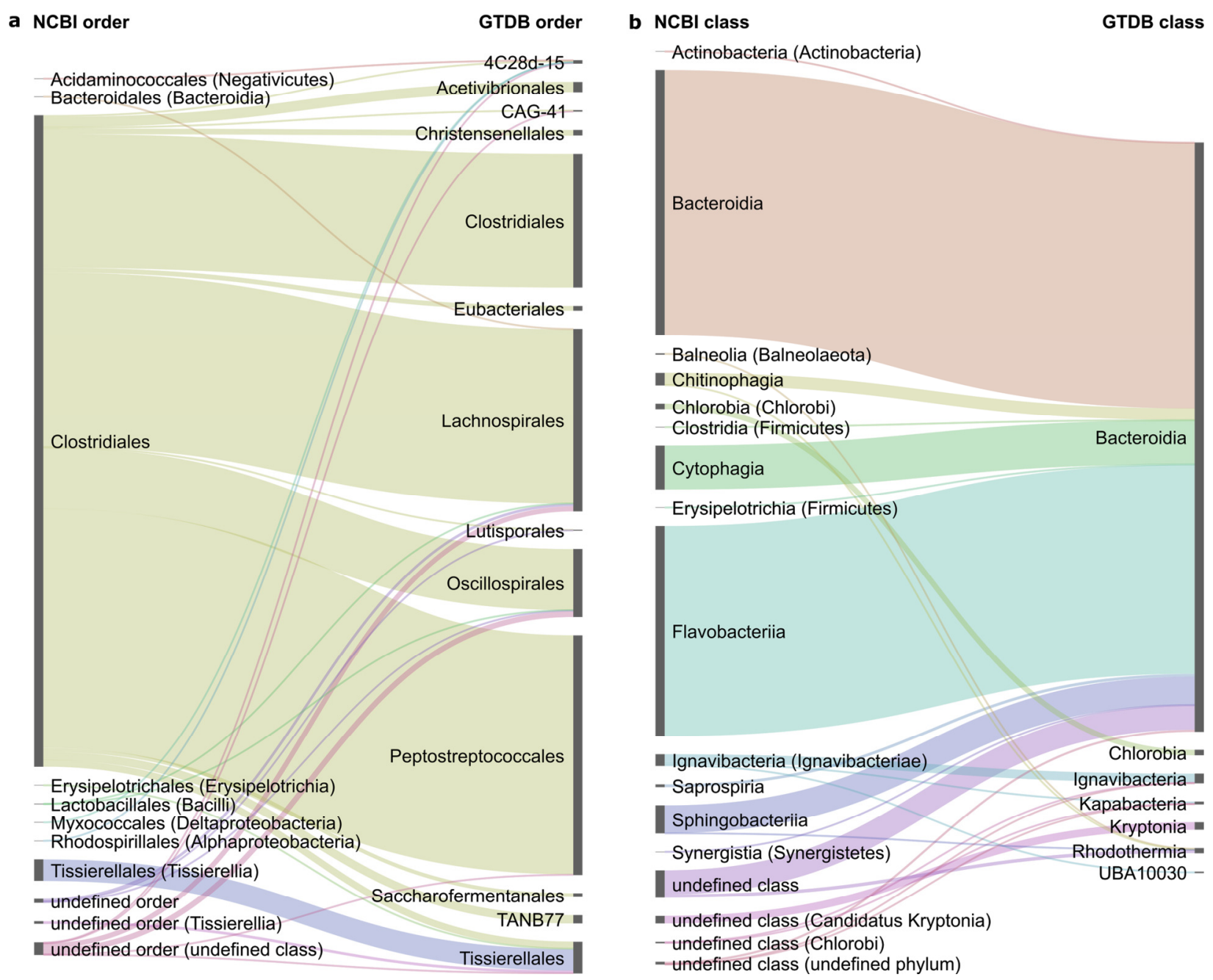

Figure 5. Comparisons of NCBI and GTDB classifications of genomes designated as 695 Clostridia or Bacteroidetes in the GTDB taxonomy. (a) Comparison of NCBI (left) and GTDB (right) order-level classifications of the 2,368 bacterial genomes assigned to the class Clostridia in the GTDB taxonomy. Genomes classified in a class other than Clostridia by NCBI are indicated in parentheses. (b) Comparison of NCBI and GTDB class-level classifications of the 2,058 bacterial genomes assigned to the phylum Bacteroidetes in the GTDB taxonomy. Genomes classified in a phylum other than the Bacteroidetes by NCBI are indicated in parentheses. 


\section{References}

705 Abbott SL and Janda JM. 2006. "The Genus Edwardsiella" in The Prokaryotes 3rd edition, eds Dworkin M et al. (New York, NY: Springer), 6: 72-89.

Anantharaman K, et al. 2016. Thousands of microbial genomes shed light on interconnected biogeochemical processes in an aquifer system. Nat Commun 7, doi: 10.1038/ncomms13219.

Beaz-Hidalgo R, Hossain MJ, Liles MR, Figueras MJ. 2015. Strategies to avoid wrongly labelled genomes using as example the detected wrong taxonomic affiliation for Aeromonas genomes in the GenBank database. PLoS One 10: e0115813.

Beiko RG. 2011. Telling the whole story in a 10,000-genome world. Biol Direct 6: 34.

Beiko RG. 2015. Microbial malaise: how can we classify the microbiome? Trends Microbiol 23: 671-679.

715 Bobay LM, Ochman H. 2017. Biological species are universal across Life's domains. Genome Biol Evol 9: 491-501.

Brown DR, in Bergey's Manual of Systematic Bacteriology. NR Krieg, W Ludwig, W Whitman, BP Hedlund, BJ Paster, JT Staley, N Ward, D Brown, A Parte, Eds. (Springer-Verlag, New York, 2010), vol. 4, pp. 567-724.

720 Brown JR, Douady CJ, Italia MJ, Marshall WE, Stanhope MJ. 2001. Universal trees based on large combined protein sequence data sets. Nat Genet 28: 281-285.

Brown CT, et al. 2015. Unusual biology across a group comprising more than $15 \%$ of domain Bacteria. Nature 523: 208-11.

Camacho C, Coulouris G, Avagyan V, Ma N, Papadopoulos J, Bealer K, Madden TL. 2009. 725 BLAST+: architecture and applications. BMC Bioinformatics 10, doi: 10.1186/1471-2105-10421.

Case RJ, Boucher Y, Dahllöf I, Holmström C, Doolittle WF, Kjelleberg S. 2007. Use of 16S rRNA and rpoB genes as molecular markers for microbial ecology studies. Appl Environ Microbiol 73: 278-288. 
730 Ciccarelli FD, Doerks T, von Mering C, Creevey CJ, Snel B, Bork P. 2006. Toward automatic reconstruction of a highly resolved tree of life. Science 311: 1283-1287.

Comas I, Homolka S, Niemann S, Gagneux S. 2009. Genotyping of genetically monomorphic bacteria: DNA sequencing in Mycobacterium tuberculosis highlights the limitations of current methodologies. PLoS One 4: e7815.

735 DeSantis TZ, Hugenholtz P, Larsen N, Rojas M, Brodie EL, Keller K, Huber T, Dalevi D, Hu P, Andersen GL. 2006. Greengenes, a chimera-checked 16S rRNA gene database and workbench compatible with ARB. Appl Environ Microbiol 72: 5069-5072.

Dupont CL, et al. 2012. Genomic insights to SAR86, an abundant and uncultivated marine bacterial lineage. ISME J 6: 1186-99.

740 Dojka MA, Hugenholtz P, Haack SK, Pace NR. 1998. Microbial diversity in a hydrocarbon- and chlorinated-solvent-contaminated aquifer undergoing intrinsic bioremediation. Appl Environ Microbiol 64: 3869-77.

Eddy SR. 2011. Accelerated Profile HMM Searches. PLoS Comput Biol 7: e1002195.

Euzeby JP. 1997. List of bacterial names with standing in nomenclature: a folder available on the internet. Int J Syst Bacteriol 47: 590-592.

Federhen S. 2012. The NCBI Taxonomy database. Nucleic Acids Res 40: D136-D143.

Finn RD, et al. 2014. Pfam: The protein families database. Nucleic Acids Res 42: D222-230.

Gadagkar SR, Rosenberg MS, Kumar S. 2005. Inferring species phylogenies from multiple genes: concatenated sequence tree versus consensus gene tree. J Exp Zool B Mol Dev Evol

750 304: 64-74.

Garrity, GM, Bell, JA, Lilburn, T, in Bergey's Manual of Systematic Bacteriology. G Garrity, DJ Brenner, NR Krieg, JT Staley, Eds. (Springer, US, 2005), vol. 2, part C, pp. 575-922.

Garrity GM, Holt JG, in Bergey's Manual of Systematic Bacteriology. G Garrity, DR Boone, RW Castenholz, Eds. (Springer-Verlag, New York, 2001), vol. 1, pp. 601-623.

755 Garrity G. 2016. A new genomics-driven taxonomy of Bacteria and Archaea: Are we there yet?. J Clin Microbiol 54: 1956-1963. 
Giovannoni SJ, Rappé MS, Vergin KL, Adair NL. 1996. 16S rRNA genes reveal stratified open ocean bacterioplankton populations related to the Green Non-Sulfur bacteria. Proc Natl Acad Sci USA 93: 7974-7984.

760 Godfray HCJ. 2002. Challenges for taxonomy. Nature 417: 17-19.

Haft DH, Selengut JD, White O. 2003. The TIGRFAMs database of protein families. Nucl Acids Res 31: 371-373.

Haft DH, et al. 2017. RefSeq: an update on prokaryotic genome annotation and curation. Nucl Acids Res: doi: 10.1093/nar/gkx1068. [Epub ahead of print].

765 Hahnke RL, Meier-Kolthoff JP, García-López M, Mukherjee S, Huntemann M, Ivanova NN, Woyke T, Kyrpides NC, Klenk HP, Göker M. 2016. Genome-based taxonomic classification of Bacteroidetes. Front Microbiol 7: 10.3389/fmicb.2016.02003.

Hug LA, et al. 2016. A new view of the tree of life. Nat Microbiol 1:

doi:10.1038/nmicrobiol.2016.48.

770 Hugenholtz P, Pitulle C, Hershberger KL, Pace NR. 1998. Novel division level bacterial diversity in a Yellowstone hot spring. J Bacteriol 180: 366-376.

Hugenholtz P, Sharshewski A, Parks DH. 2016. Genome-based microbial taxonomy coming of age. In Microbial Evolution (ed. Ochman, H.) 55-65 (Cold Spring Harbor Laboratory Press, Cold Spring Harbor, New York, USA.

775 Hyatt D, Chen GL, Locascio PF, Land ML, Larimer FW, Hauser LJ. 2010. Prodigal: Prokaryotic gene recognition and translation initiation site identification. BMC Bioinformatics 11: 119.

Janda JM, Abbott SL. 2007. 16S rRNA gene sequencing for bacterial identification in the diagnostic laboratory: pluses, perils, and pitfalls. J Clin Microbiol 45: 2761-2764.

Jumas-Bilak E, Roudière L, Marchandin H. 2009. Description of 'Synergistetes' phyl. nov. and emended description of the phylum 'Deferribacteres' and of the family Syntrophomonadaceae, phylum 'Firmicutes'. Int J Syst Evol Microbiol 59: 1028-1035.

Kook JK, Park SN, Lim YK, Cho E, Jo E, Roh H, Shin Y, Paek J, Kim HS, Kim H, Shin JH, Chang YH. 2017. Genome-based reclassification of Fusobacterium nucleatum subspecies at the species level. Curr Microbiol 74: 1137-1147. 
785 Konstantinidis KT and Tiedje JM. 2005. Towards a genome-based taxonomy for prokaryotes. $J$ Bacteriol 187: 6258-6264.

Konstantinidis KT Rosselló-Móra R, Amann R. 2017. Uncultivated microbes in need of their own taxonomy. ISME J 11: 2399-2406. doi: 10.1038/ismej.2017.113

Kumar S, Stecher G, Suleski M, Hedges SB. 2017. TimeTree: A resource for timelines, timetrees, and divergence times. Mol Biol Evol 34: 1812-1819.

Lang JM, Darling AE, Eisen JA. Phylogeny of bacterial and archaeal genomes using conserved genes: supertrees and supermatrices. PLoS One 8: doi: 10.1371/journal.pone.0062510.

Lawson PA, Citron DM, Tyrrell KL, Finegold SM. 2016. Reclassification of Clostridium difficile as Clostridioides difficile (Hall and O'Toole 1935) Prévot 1938. Anaerobe 40: 95-99.

795 Le Si Q, Gascuel O. 2008. An improved general amino-acid replacement matrix. Mol Bio Evol 25: $1307-1320$.

Leinonen R, Sugawara H, Shumway M. 2011. The Sequence Read Archive. Nuc Acids Res 39: D19-D21.

Lonergan DJ, Jenter HL, Coates JD, Phillips EJP, Schmidt TM, Lovley DR. 1996. Phylogenetic analysis of dissimilatory Fe(III)-reducing bacteria. J Bacteriol 178: 2402-2408.

Ludwig W, Euzeby J, Whitman WB, in Bergey's Manual of Systematic Bacteriology. WB Whitman, M Goodfellow, P Kämpfer, HJ Busse, M Trujillo, W Ludwig, K Suzuki, A Parte, Eds. (Springer-Verlag, New York, 2012), vol. 5, part B, pp. 2000-2001.

Ludwig W, Klenk HP, in Bergey's Manual of Systematic Bacteriology. G Garrity, Ed. (Springer, US, 2001), vol. 2, part A, pp. 49-66.

Ludwig W, Strunk O, Westram R, Richter L, Meier H, Yadhukumar, Buchner A, Lai T, Steppi S, Jobb G et al. 2004. ARB: a software environment for sequence data.. Nucleic Acids Res 32: $1363-1371$.

Maniloff J, in Molecular Biology and Pathogenicity of Mycoplasma. S Razin, R Herrmann, Eds. (Springer, US, 2002), pp 31-43. 
Marin J, Battistuzzi FU, Brown AC, Hedges SB. 2016. The timetree of Prokaryotes: New insights into their evolution and speciation. Mol Biol Evol 34: 437-446.

Martiny JBH, Bohannan BJM, Brown JH, Colwell RK, Fuhrman JA, Green JL, Horner-Devine MC, Kane M, Krumins JA, Kuske CR, Morin PJ, Naeem S, Øvreås L, Reysenbach AL, Smith VH, Staley JT. 2006. Microbial biogeography: Putting microorganisms on the map. Nat Rev Microbiol 4: 102-112.

McDonald D, Price MN, Goodrich J, Nawrocki EP, DeSantis TZ, Probst A, Andersen GL, Knight R, Hugenholtz P. 2012. An improved Greengenes taxonomy with explicit ranks for ecological and evolutionary analyses of bacteria and archaea. ISME J 6: 610-618.

Munoz R, Rosselló-Móra R, Amann R. 2016. Revised phylogeny of Bacteroidetes and proposal of sixteen new taxa and two new combinations including Rhodothermaeota phyl. nov. Syst Appl Microbiol 39: 281-296.

Nawrocki EP, Ph.D thesis. Structural RNA Homology Search and Alignment using Covariance Models. Washington University in Saint Louis (2009).

825 Ondov BD, et al. 2016. Mash: Fast genome and metagenome distance estimation using MinHash. Genome Biol 17: 132.

Oren A, da Costa MS, Garrity GM, Rainey FA, Rosselló-Móra R, Schink B, Sutcliffe I, Trujillo ME, Whitman WB. 2015. Proposal to include the rank of phylum in the International Code of Nomenclature of Prokaryotes. Int J Syst Evol Microbiol 65: 4284-4287.

830 Parker CT, Tindall BJ, Garrity GM. 2015. International Code of Nomenclature of Prokaryotes. Int J Syst Evol Microbiol doi:10.1099/ijsem.0.000778.

Parks DH, Imelfort M, Skennerton CT, Hugenholtz P, and Tyson GW. 2015. CheckM: assessing the quality of microbial genomes recovered from isolates, single cells, and metagenomes. Genome Res 25: 1043-1055.

835 Parks DH, Rinke C, Chuvochina M, Chaumeil PA, Woodcroft BJ, Evans PN, Hugenholtz P, Tyson GW. 2017. Recovery of nearly 8,000 metagenome-assembled genomes substantially expands the tree of life. Nat Microbiol 2: 1533-42. 
Podosokorskaya OA, et al. 2013. Characterization of Melioribacter roseus gen. nov., sp. nov., a novel facultatively anaerobic thermophilic cellulolytic bacterium from the class

$840 \quad$ Ignavibacteria, and a proposal of a novel bacterial phylum Ignavibacteriae. Environ Microbiol 15: 1759-1771.

Price MN, Dehal PS, and Arkin AP. 2009. Fasttree: Computing large minimum evolution trees with profiles instead of a distance matrix. Mol Biol Evol 26: 1641-1650.

Quast C, Pruesse E, Yilmaz P, Gerken J, Schweer T, Yarza P, Peplies J, Glöckner FO. 2013. The 845 SILVA ribosomal RNA gene database project: Improved data processing and web-based tools. Nucleic Acids Res 41: D590-596.

Rinke C, et al. 2013. Insights into the phylogeny and coding potential of microbial dark matter. Nature 499: 431-437.

Sakamoto M, Iino T, Ohkuma M. 2017. Faecalimonas umbilicata gen. nov., sp. nov., isolated from human faeces, and reclassification of Eubacterium contortum, Eubacterium fissicatena and Clostridium oroticum as Faecalicatena contorta gen. nov., comb. nov., Faecalicatena fissicatena comb. nov. and Faecalicatena orotica comb. nov. Int J Syst Evol Microbiol 67: 1219-1227.

Schulz F, Eloe-Fadrosh EA, Bowers RM, Jarett J, Nielsen T, Ivanova NN, Kyrpides NC, Woyke 855 T. 2017. Towards a balanced view of the bacterial tree of life. Microbiome 5: 140.

Segata N, Börnigen D, Morgan XC, Huttenhower C. 2013. PhyloPhlAn is a new method for improved phylogenetic and taxonomic placement of microbes. Nat Commun 4: doi:10.1038/ncomms3304.

Sekiguchi Y, Ohashi A, Parks DH, Yamauchi T, Tyson GW, Hugenholtz P. 2015. First genomic 860 insights into members of a candidate bacterial phylum responsible for wastewater bulking. PeerJ 3: e740.

Skennerton CT, et al. 2016. Phylogenomic analysis of Candidatus 'Izimaplasma' species: freeliving representatives from a Tenericutes clade found in methane seeps. ISME J 10: 26792692.

865 Tanner MA, Everett CL, Coleman WJ, Yang MM. 2000. Complex microbial communities inhabiting sulfide-rich black mud from marine coastal environments. Biotechnology et alia $\mathbf{8}$ : 1-16. 
Thiergart T, Landan G, Martin WF. 2014. Concatenated alignments and the case of the disappearing tree. BMC Evol Biol 14: doi: 10.1186/s12862-014-0266-0.

870 Tonini J, Moore A, Stern D, Shcheglovitova M, Ortí G. 2015. Concatenation and Species Tree Methods Exhibit Statistically Indistinguishable Accuracy under a Range of Simulated Conditions. PLoS Curr 7: doi: 10.1371/currents.tol.34260cc27551a527b124ec5f6334b6be.

Trost B, Haakensen M, Pittet V, Ziola B, Kusalik A. 2010. Analysis and comparison of the pangenomic properties of sixteen well-characterized bacterial genera. BMC Microbiol 10: 258. doi: 10.1186/1471-2180-10-258.

Vanwonterghem I, Jensen PD, Rabaey K, Tyson DW. 2016. Genome-centric resolution of microbial diversity, metabolism and interactions in anaerobic digestion. Environ Microbiol 18: $3144-3158$.

Waite DW, et al. 2017. Comparative Genomic Analysis of the Class Epsilonproteobacteria and Proposed Reclassification to Epsilonbacteraeota (phyl. nov.). Front Microbiol 8: 10.3389/fmicb.2017.00682.

Whelan S, Goldman N. 2001. A general empirical model of protein evolution derived from multiple protein families using a maximum-likelihood approach. Mol Biol Evol 18: 691-699.

Whitman WB. 2016. Modest proposals to expand the type material for naming of prokaryotes. Int J Syst Evol Microbiol 66: 2108-2112.

Williams TA, Heaps SE, Cherlin S, Nye TMW, Boys RJ, Embley TM. 2015. New substitution models for rooting phylogenetic trees. Philos Trans R Soc Lond B Biol Sci 370: 20140336.

Wolf M, Müller T, Dandekar T, Pollack JD. 2004. Phylogeny of Firmicutes with special reference to Mycoplasma (Mollicutes) as inferred from phosphoglycerate kinase amino acid sequence data. Int J Syst Evol Microbiol 54: 871-875.

Wu D, Jospin G, Eisen JA. 2013a. Systematic identification of gene families for use as "markers" for phylogenetic and phylogeny-driven ecological studies of bacteria and archaea and their major subgroups. PLoS One 8: e77033.

Wu D, Doroud L, Eisen JA. 2013b. TreeOTU: Operational Taxonomic Unit Classification Based on Phylogenetic Trees. arXiv:1308.6333 [q-bio.GN]. 
Yamada Y, et al. 2007. Characterization of filamentous bacteria, belonging to candidate phylum KSB3, that are associated with bulking in methanogenic granular sludges. ISME J 1: 246-255.

900 Yang Z. 1994. Maximum likelihood phylogenetic estimation from DNA sequences with variable rates over sites: approximate methods. J Mol Evol 39: 306-314.

Yarza P, Richter M, Peplies J, Euzeby J, Amann R, Schleifer KH, Ludwig W, Glöckner FO, and Rossello-Mora R. 2008. The All-Species Living Tree project: A 16S rRNA-based phylogenetic tree of all sequenced type strains. Syst Appl Microbiol 31: 241-250.

905 Yarza P, et al. 2014. Uniting the classification of cultured and uncultured bacteria and archaea using 16S rRNA gene sequences. Nat Rev Microbiol 12: 635-645.

Yilmaz P, Parfrey LW, Yarza P, Gerken J, Pruesse E, Quast C, Schweer T, Peplies J, Ludwig W, Glöckner FO. 2014. The SILVA and "All-species Living Tree Project (LTP)" taxonomic frameworks. Nucl. Acids Res 42: D643-D648.

910 Yoon SH, Ha SM, Kwon 1, Lim J, Kim Y, Seo H, Chun J. 2017. Introducing EzBioCloud: a taxonomically united database of 16S rRNA gene sequences and whole-genome assemblies. Int J Syst Evol Microbiol 67: 1613-1617.

Yutin N, Galperin MY. 2013. A genomic update on clostridial phylogeny: Gram-negative spore formers and other misplaced clostridia. Environ Microbiol 15: 2631-2641.

915 Zhang Y, Sievert SM. 2013. Pan-genome analyses identify lineage- and niche-specific markers of evolution and adaptation in Epsilonproteobacteria. Front Microbiol 5: 110.

Zwart G, van Hannen EJ, Kamst-van Agterveld MP, Van der Gucht K, Lindström ES, Van Wichelen J, Lauridsen T, Crump BC, Han SK, Declerck S. 2003. Rapid screening for freshwater bacterial groups by using reverse line blot hybridization. Appl Environ Microbiol

$920 \quad$ 69: $5875-5883$. 